International Journal of Advanced Trends in Computer Science and Engineering

Available Online at http://www.warse.org/IJATCSE/static/pdf/file/ijatcse691032021.pdf

https://doi.org/10.30534/ijatcse/2021/691032021

\title{
ECG Arrhythmia Time Series Classification Using 1D Convolution -LSTM Neural Networks
}

\author{
Yousra MjAlqaisi ${ }^{1}$, Muhammad Aziz Muslim ${ }^{2}$, Rahmadwati ${ }^{3}$ \\ ${ }^{1}$ University of Brawijaya, Palestine, alqaisiyusra@gmail.com \\ ${ }^{2}$ University of Brawijaya, Indonesia, muh_aziz@ub.ac.id \\ ${ }^{3}$ University of Brawijaya, Indonesia, rahma@ub.ac.id
}

\begin{abstract}
An electrocardiogram (ECG) can be dependably used as a measuring device to monitor cardiovascular function. The abnormal heartbeat appears in the ECG pattern and these abnormal signals are called arrhythmias. Classification and automatic arrhythmia signals can provide a faster and more accurate result. Several machine learning approaches have been applied to enhance the accuracy of results and increase the speed and robustness of models. This paper proposes a method based on Time-series Classification using deep Convolutional -LSTM neural networks and Discrete Wavelet Transform to classify 4 different types of Arrhythmia in the MIT-BIH Database. According to the results, the suggested method gives predictions with an average accuracy of $97 \%$ without needing to do feature extraction or data augmentation.
\end{abstract}

Key words:Convolution neural network, Deep Learning, ECG signals classification, Wavelet transforms.

\section{INTRODUCTION}

Cardiovascular disease is the number one cause of death globally, taking around 17.9 million people each year and accounting for $31 \%$ of all global deaths[1]. In general, there are three large groups of cardiovascular diseases: electrical (arrhythmias), structural (Cardiomyopathy), blood circulation (vascular disorder), and [2], [3]. This paper focused on arrhythmia.

The electrical system that regulates the heartbeat is stable and generates two kinds of beats normal heartbeats and an abnormal heartbeat called an arrhythmia. The basis for diagnosing arrhythmias is the determination of normal versus abnormal heart rhythms. A standard tool to diagnose arrhythmias is the electrocardiogram (ECG): measurement of the heart's electrical activity, as shown in Figure 1, the ECG waveform includes five main waves of $\mathrm{P}, \mathrm{Q}, \mathrm{R}, \mathrm{S}$, and $\mathrm{T}$ [4].

The equipment to produce electrocardiograms is principally present in hospitals, where cardiologists use their knowledge and experience to carefully evaluate the ECG.

However, this activity is time-consuming and prone to error, so an automatic approach can support them during their decision that aims to reduce subjectivity.
Recent literature focuses on classification; various authors have tested ECG arrhythmia classification using different ways such as statistical methods, expert systems, support vector machines, and supervised neural networks [5-10].

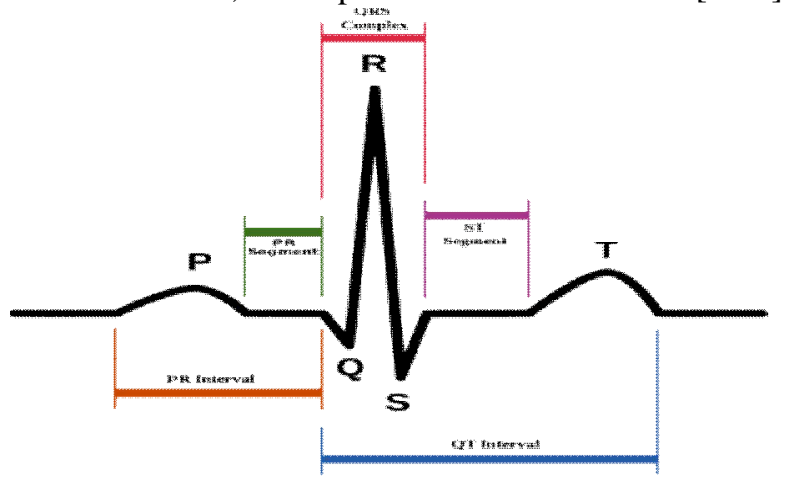

Figure 1:ECG waves

\section{DATA SOURCE AND COLLECTION}

The Massachusetts Institute of Technology (MIT-BIH) Arrhythmia Database is used to classify the beats of the ECG [11], its available on the PhysioNet website contains 48 patient ECG records, every 30 minutes Lead II was used to record most of the ECG signals[12]. Data studied by the Arrhythmia Laboratory BIH between 1975 and 1979.

The participants were 25 men and 22 women between the ages of 22 and 89 . The dataset is accompanied by annotation files that aid in finding the beats as usual or arrhythmias. The recordings are obtained with a frequency of $360 \mathrm{~Hz}$ and applied bandpass filter at $0.1-100 \mathrm{~Hz}$.

Two or more cardiologists annotated each record, each annotation consistent with the $\mathrm{R}$ peak of a single beat so that we have implicitly solved for this specific case the beat detection problem.

\subsection{ECG heartbeats}

According to the Association for the Advancement of Medical Devices (AAMI) (2012), arrhythmia can be classified into five major categories: normal, supraventricular ectopic beats, ventricular ectopic beats, fusion beats, and unknown beats [13]. 
The beats are classified into 12 different types, each represented in database records by a letter. In this study, we choose 4 types 'N',' R', ' V', ' F', to classify them. as shown in Table 1 the four arrhythmia classes were chosen for this study.

Table 1: The four heartbeats classified in the study

\begin{tabular}{|c|c|c|c|}
\hline Class & $\begin{array}{c}\text { Training } \\
\text { Data }\end{array}$ & $\begin{array}{c}\text { Validation } \\
\text { data }\end{array}$ & $\begin{array}{c}\text { \# of each } \\
\text { class in all } \\
\text { database }\end{array}$ \\
\hline N & 2000 & 14000 & 75011 \\
\hline R & 2000 & 1420 & 7255 \\
\hline V & 2000 & 1460 & 7129 \\
\hline F & 1420 & 361 & 1784 \\
\hline
\end{tabular}

\subsection{The Dataset Upsample}

According to the dataset, the $\mathrm{N}$ class is more populated than the others: it constitutes $89 \%$ of the whole dataset. This overbalancing can lead to inconsistent training and poor quality algorithms. To solve this we do an up-sample by duplicate the data. We have considered two dataset configurations. In the first one, we have built the test set; second, we have built the Validation set, by randomly choosing from the dataset.

Note that 2000 samples are randomly sampled of each category for training and validation (90:10)\% respectively, as shown in Table 2 that class F contains only 1784 total samples so it's up-sampled to 2000 samples by random repeating.

As shown in Figures 2 and 3 the frequency distribution for the different labels before and after the up-sampling approach.

Table 2:Numbers of beats in each class

\begin{tabular}{|c|c|c|}
\hline Class & Name & Definition \\
\hline $\mathrm{N}(0)$ & Normal beat & Normal \\
\hline $\mathrm{R}(1)$ & $\begin{array}{c}\text { Right bundle } \\
\text { branch block beat }\end{array}$ & $\begin{array}{c}\text { Happened when a } \\
\text { block in the right } \\
\text { bundle of the } \\
\text { electrical conduction } \\
\text { system in the heart. }\end{array}$ \\
\hline $\mathrm{V}(2)$ & $\begin{array}{c}\text { Premature } \\
\text { ventricular } \\
\text { contraction }\end{array}$ & $\begin{array}{c}\text { Happened where the } \\
\text { heartbeat is initiated } \\
\text { by Purkinje fibers in } \\
\text { the ventricles rather } \\
\text { than by the SA node }\end{array}$ \\
\hline $\mathrm{F}(3)$ & Fusion & $\begin{array}{c}\text { Fusion beats occur } \\
\text { when electrical } \\
\text { impulses from } \\
\text { different sources act } \\
\text { on the same area }\end{array}$ \\
\hline
\end{tabular}

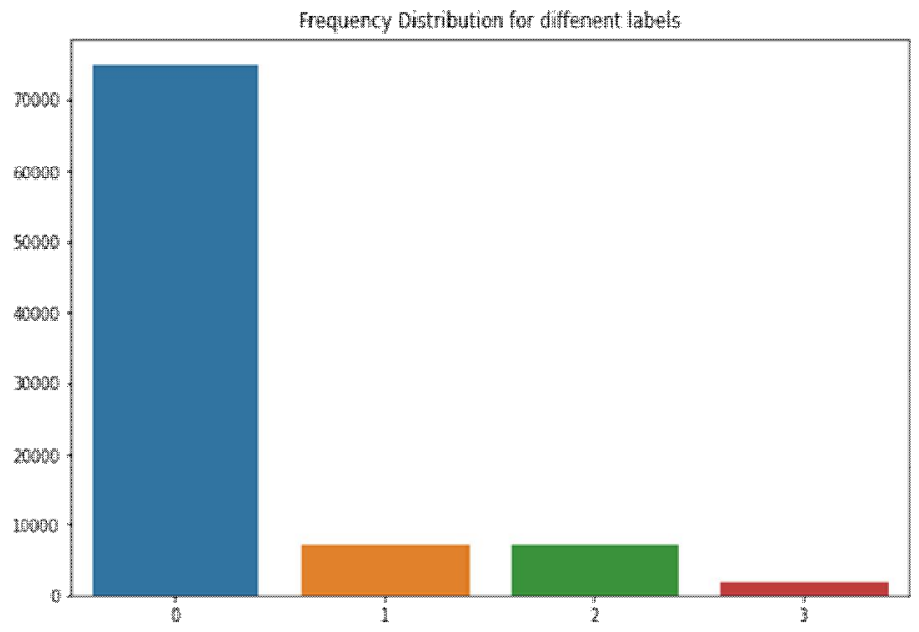

Figure 2:Frequency Distribution For The Different Labels Before The Upsampling Approach

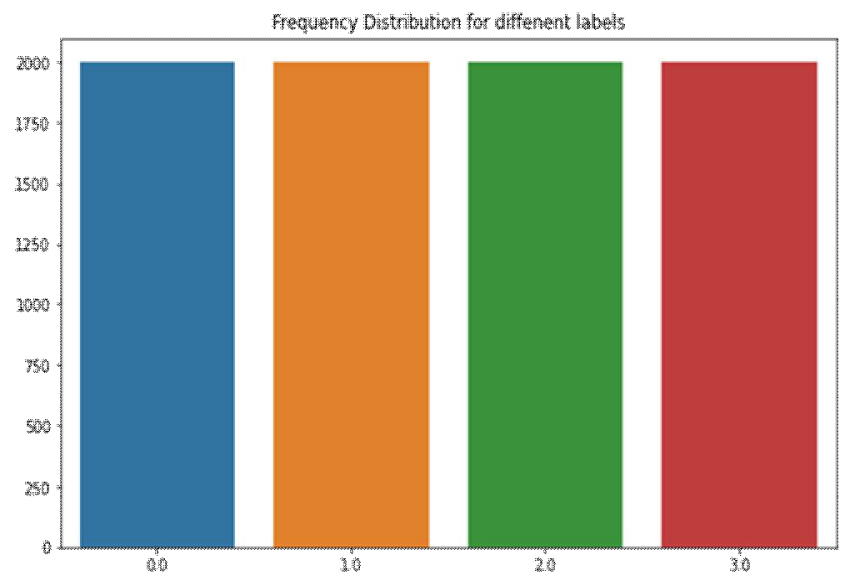

Figure 3: Frequency Distribution For The Different Labels After The Upsampling Approach

\section{METHODOLOGY}

The System WorkFlow is shown in Figure 4, starts with the ECG signal that we will use from the MIT-BIH database and select the four beats type as we defined in this study after that we will define $\mathrm{R}$ peaks according to data files annotation using the native Python waveform-database (WFDB) package [14].

Then, a window surrounded R- peak will be taken. Discrete wavelet transforms will be applied for each pulse of ECG signal. For normalization, Min-MaxScale is used. 


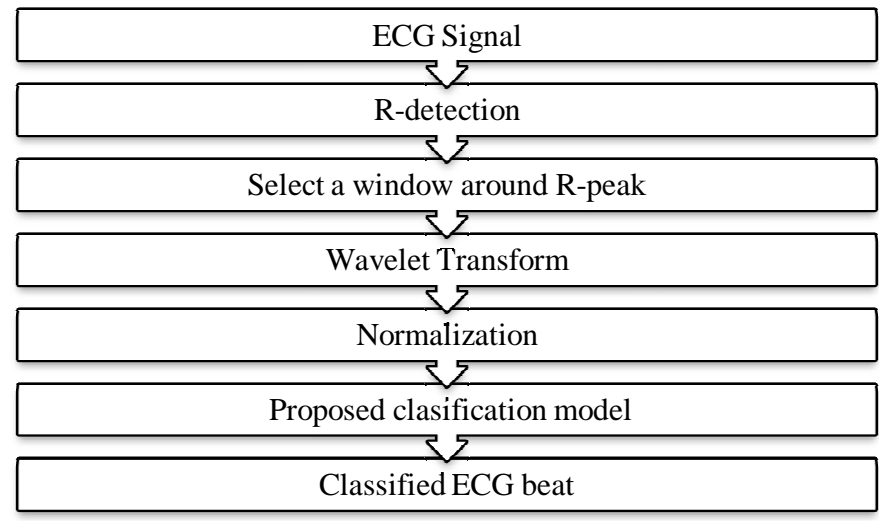

Figure 4: System WorkFlow

\subsection{Data preparation}

Before the evaluation of model, the data preparation as follows :

1) Splitting the ECG signal from continuous signal to single beat (pulses) as shown in Figure 5 every class has its different signal.

2) $R$ detection, using the data files annotation using (WFDB) package.

3) Select the window around $R$ in this research for each record; we will select the window size one second: $\quad-500 \mathrm{~ms}$ to $+500 \mathrm{~ms}$ around the R-peak.

4) Discrete wavelet transform.

\subsection{Wavelet Transform}

The ECG signal is non-stationary because the heart generates it under the influence of physiological factors that depend on the brain and constantly change over time. So the Wavelet Transform is applied specially Discrete wavelet transforms of Symlet 4 Family, every category had composed of four different frequency levels that will make the deep learning model work effectively.

Continually, the amplitude values were normalized to the range of between zero and one.

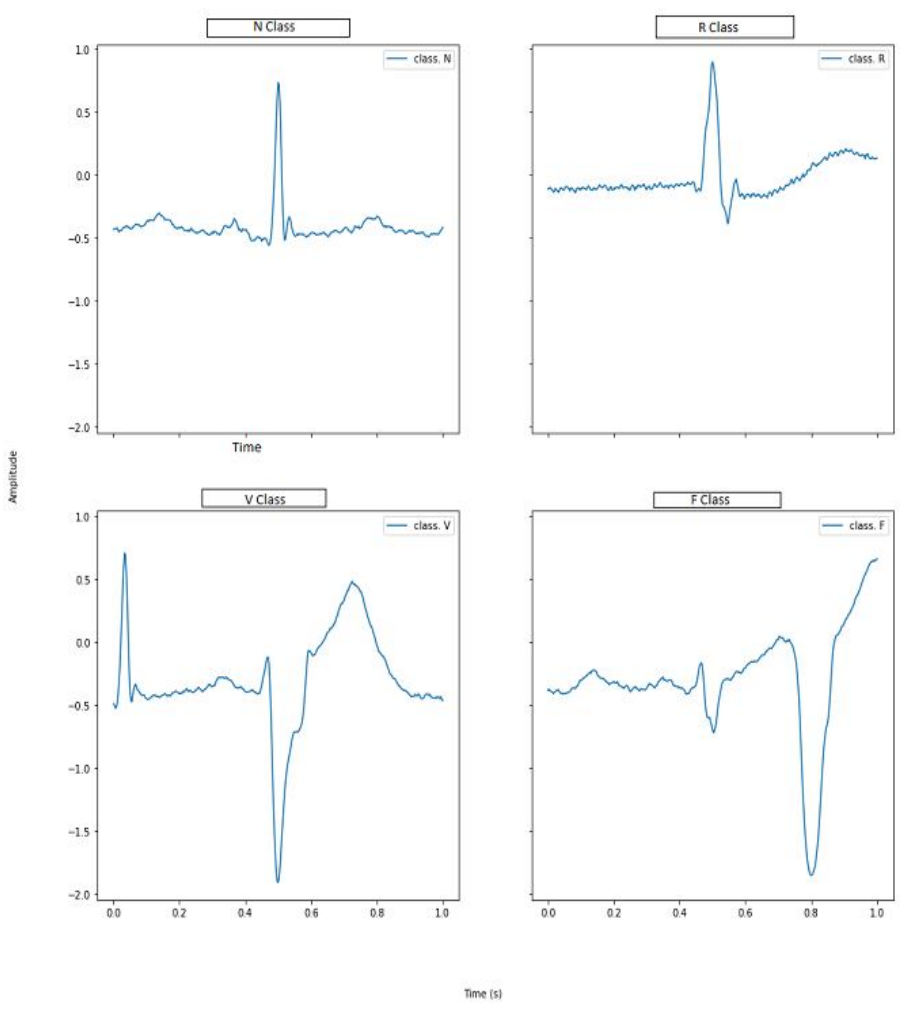

Figure 5: The Four Classes that Used In The Model

\subsection{Proposed Deep Learning Model}

This paper proposed to use two 1D convolution layers and an LSTM layer, then the outputs will be taken by two Dense layers. Figure 6 depicts the proposed model.

In Figure 6, the first one from 1D CNN has 32 filters and kernel size 15; and the second one 1D CNN has 64 filters and kernel size 5, LSTM layer with 256 units (neuron), these three layers used the ReLU as Activation Function.

By using 64 filters in the second layer of 1D CNN we provide convergence stability to the model, other researchers used 32 filters and kernel size 5 in their model[15].

The activation function ReLU is Rectified Linear Unit is used in this study. The neural networks used it to decide the output by mapping output to some values like 1 and 0 , depending on the model function.

The fully connected layers are two dense, the first dense layer with 64 units and ReLU activation function, and the second dense layer with 4 units and Softmax activation layer, which aims to predict the output class probabilities.

The Dropout layers have a rate of 0.5 . 


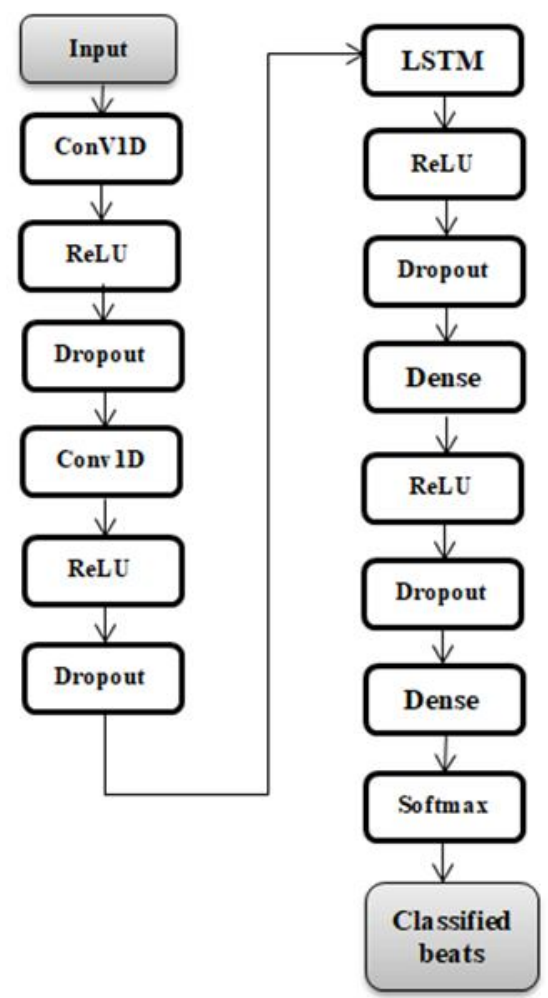

Figure 6: The Architecture Of The Proposed Networks

\subsection{Implementation}

The model has been trained on Google Colaboratory [19], and we use the deep learning libraries to implement the model as Keras[20] and Tensor Flow[21] for Python 3.6 [22] wherein the proposed model is trained for 50 epochs.

The Softmax output is the loss function from cross-entropy loss. We use in this model Adam optimizer [23] with a learning rate of 0.0001 that decays exponentially by a factor of 0.1 .

\subsection{Evaluate the performance of classification}

In order to evaluate the performance of classification including precision, recall and F-score.Here the Classification Report on the training data, as it's shown in Figure 8 the performance is excellent, F1 score and Recall are almost 1 .

1. Precision is the fraction of true positive examples among the number of false positives plus true positives.

$$
\text { precision }=\frac{t p}{t p+f p}(1)
$$

2. Recall also referred to as "sensitivity, is the fraction of the number of true positives among the number of true positives plus false negatives).

$$
\text { recall }=\frac{t p}{t p+f n}(2)
$$

If the Precision and Recall both equal to 1 then we said that the classifier is perfect.

3. The F1-score is a measure of a model's accuracy on a dataset.

A perfect model has an F-score of 1 .

$$
F 1=\frac{t p}{t p+\frac{1}{2}(f p+f n)}
$$

Where :

tp The number of true positives values that are classified by the model.

fn The number of false negatives values that are classified by the model.

fp The number of false positives values that are classified by the model.

\section{RESULTS}

The evaluation of arrhythmia classifier in Section 3.3 has been applied on 8000 heartbeats (2000 from each class).

In Figure 7 which is the confusion matrix for the applied classifier, it's clear that the model can make accurate classification and distinguish between the different classes with overall accuracy in the training set is $\mathbf{9 9 \%}$. 


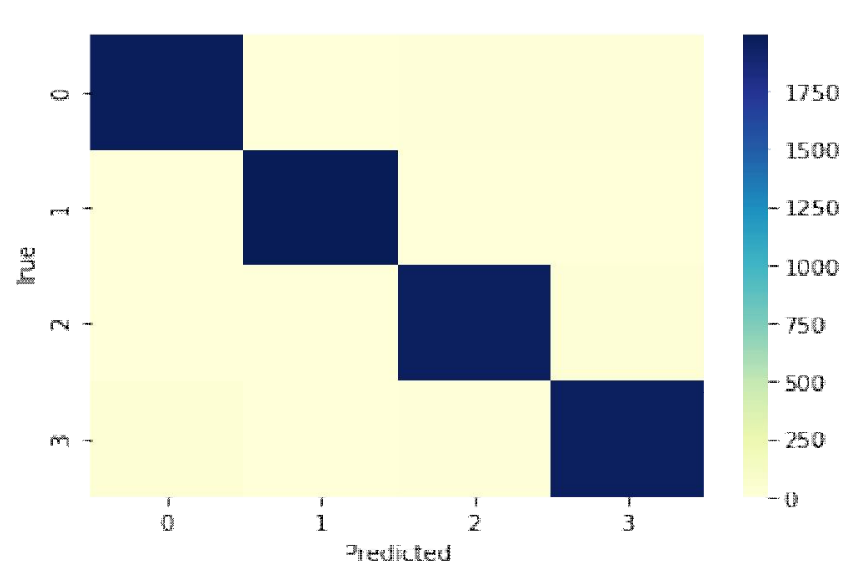

Figure 7: The Confusion Matrix For Applying The Model On The Training Set

$\begin{array}{rrrrr} & \text { precision } & \text { recall } & \text { f1-score } & \text { support } \\ 0 & 0.98 & 0.99 & 0.98 & 2000 \\ 1 & 1.00 & 1.00 & 1.00 & 2000 \\ 2 & 0.99 & 0.98 & 0.98 & 2000 \\ 3 & 0.98 & 0.98 & 0.98 & 2000 \\ & & & & \\ \text { accuracy } & & & 0.99 & 8000 \\ \text { macro avg } & 0.99 & 0.99 & 0.99 & 8000 \\ \text { weighted avg } & 0.99 & 0.99 & 0.99 & 8000\end{array}$

Figure8: The Classification Report On The Training Data

We get $\mathbf{9 7 \%}$ accuracy when we evaluated the arrhythmia classifier of Section 3.3 on 18236 heartbeats. The confusion matrix in Figure 9 appears that the model gives accurate predictions and classifying four different classes when applying the classifier on the testing set.

Also, the Classification Report in Figure10 shows that the performance of testing data is very good.

F1score and Recall are close to $\boldsymbol{I}$ which is the ideal number for them.

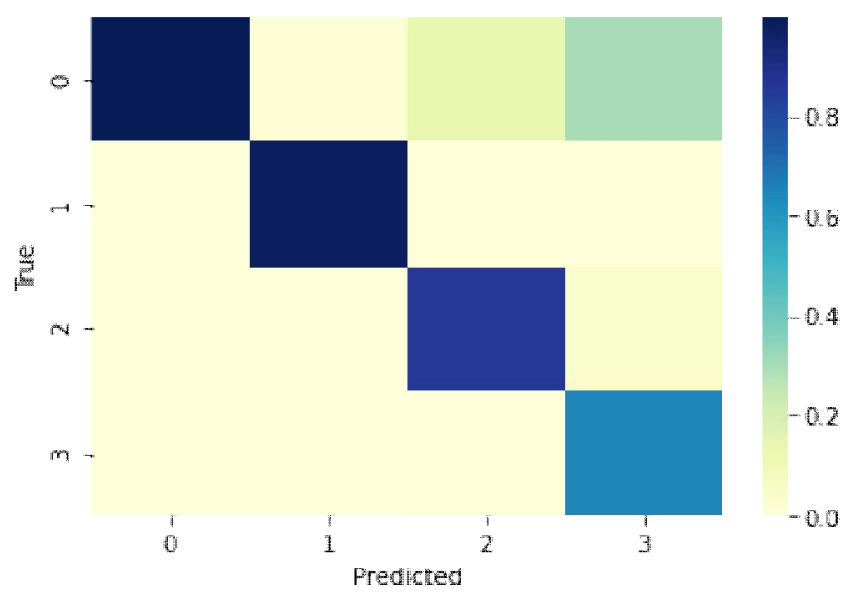

Figure 9: The Confusion Matrix For Applying The Model On The Testing Set

$\begin{array}{rrrrr} & \text { precision } & \text { recall } & \text { f1-score } & \text { support } \\ 0 & 1.00 & 0.97 & 0.99 & 14990 \\ 1 & 0.98 & 0.99 & 0.99 & 1422 \\ 2 & 0.86 & 0.98 & 0.91 & 1461 \\ 3 & 0.65 & 0.94 & 0.77 & 363 \\ & & & & \\ \text { accuracy } & & & 0.97 & 18236 \\ \text { macro avg } & 0.87 & 0.97 & 0.91 & 18236 \\ \text { weighted avg } & 0.98 & 0.97 & 0.98 & 18236\end{array}$

Figure 10: The Classification Report On The Testing Data

Table 3 shows a comparison of heartbeat classification results between this paper and some others research.

Table 3: Comparison of heartbeat classification results

\begin{tabular}{|c|c|c|}
\hline Work & Approach & $\begin{array}{c}\text { Average } \\
\text { Accuracy } \\
\text { (\%) }\end{array}$ \\
\hline This paper & $\begin{array}{c}\text { CNN-LSTM } \\
\text { +DWT }\end{array}$ & $\mathbf{9 7}$ \\
\hline $\begin{array}{c}\text { M. Kachuee et } \\
\text { al.[15] }\end{array}$ & $\begin{array}{c}\text { Deep residual CNN } \\
\text { Martis et al.[16] }\end{array}$ & 93.4 \\
\hline DWharya et al.[17] & $\begin{array}{c}\text { Augmentation + } \\
\text { CNN }\end{array}$ & 93.8 \\
\hline Li et al.[18] & $\begin{array}{c}\text { DWT + random } \\
\text { forest }\end{array}$ & 93.5 \\
\hline
\end{tabular}




\section{REFERENCES}

1. World Health Organization, "Cardiovascular diseases (CVDs),'2017.[Online].

Available: https://www.who.int/en/news-room/factsheets/detail/cardiovascular-diseases-(cvds)

2. Heart Rhythm Society, "Heart diseases and disorders," 2017.[Online].

Available: https://upbeat.org/heart-rhythm-disorders.

3. Texas Heart Institute, "Categories of arrhythmias," 2016.[Online].

Available:https://www.texasheart.org/heart-health/heartinformation-center/topics/categories-of-arrhythmias/.

4. National Heart Lung and Blood Institute, "Electrocardiogram," 2016. [Online].

Available:https://www.nhlbi.nih.gov/healthtopics/electrocardiogram.

5. Mustaqeem, A.; Anwar, S.M.; Khan, A.R.; Majid, M. A statistical analysis based recommender model for heart disease patients. Int. J. Med. Inform. 2017, 108, 134-145.

6. V.Goutham, SaiKrishna, ShruthiKovela. A Study of incremental Learning model using deep neural network, International Journal of Advanced Trends in Computer Science and Engineering, vol. 10,no.2,March-April 2021

7. Hu, Y.H.; Palreddy, S.; Tompkins, W.J. A patientadaptable ECG beat classifier using a mixture of experts approach. IEEE Trans. Biomed. Eng. 1997, 44, 891-900.

8. Hu, Y.H.; Tompkins, W.J.; Urrusti, J.L.; Afonso, V.X. Applications of artificial neural networks for ECG signal detection and classification. J. Electrocardiol. 1993, 26, 66-73

9. Salem, M.; Taheri, S.; Yuan, J.S. ECG arrhythmia classification using transfer learning from 2dimensional deep CNN features. InProceedings of the 2018 IEEE Biomedical Circuits and Systems Conference (BioCAS), Cleveland, OH, USA, 17-19 October 2018; IEEE: Piscataway, NJ, USA, 2018; pp. $1-4$

10. Osowski, S.; Hoai, L.T.; Markiewicz, T. Support vector machine based expert system for reliable

heartbeat recognition.IEEE Trans. Biomed. Eng. 2004, 51, 582-589.

11. Moody, G.B.; Mark, R.G. The impact of the MITBIH arrhythmia database. IEEE Eng. Med. Biol. Mag. 2001, 20, 45-50.

12. https://www.physionet.org/content/mitdb/1.0.0/

13. American National Standards Institute, "Testing and reporting performance results of cardiac rhythm and ST segment measurement algorithms," 2012.

14. https://archive.physionet.org/physiotools/wfdb.shtml

15. M. Kachuee, S. Fazeli et al., "ECG Heartbeat Classification: A Deep Transferable Representation", 2018 IEEE International Conference on Healthcare Informatics (ICHI). [Online].Available: https://arxiv.org/abs/1805.00794

16. R. J. Martis, U. R. Acharya, K. M. Mandana, et al, "Cardiac decision making using higher order spectra". Biomedical Signal Processing and Control, vol. 8, no. 2, pp.193-203, 2013

17. U. R. Acharya, S. L. Oh, Y. Hagiwara, J. H. Tan, M. Adam, A. Gertych, and R. San Tan, "A deep convolutional neural network model to classify heartbeats," Computers in biology and medicine, vol. 89, pp. 389-396, 2017

18. T. Li and M. Zhou, "Ecg classification using wavelet packet entropy and random forests," Entropy, vol. 18, no. 8, p. 285, 2016.

19. GoogleColaboratory, https://colab.research.google.com/

20. Keras, https://github.com/keras-team/keras/

21. M. Abadi, A. Agarwal, P. Barham, E. Brevdo, Z. Chen, C. Citro, G. S. Corrado, A. Davis, J. Dean, M. Devin et al., "Tensorflow: Large-scale machine learning on heterogeneous distributed systems," arXiv preprint arXiv:1603.04467, 2016

22. Python3.6, https://www.python.org/downloads/release/python-360/

23. D. Kingma and J. Ba, "Adam: A method for stochastic optimization," arXiv preprint arXiv:1412.6980, 2014. 